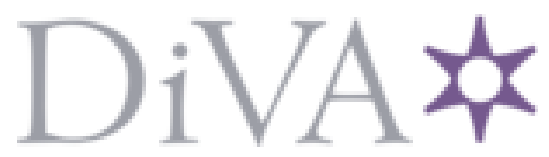

http://www.diva-portal.org

This is the published version of a paper published in Physical Review B. Condensed Matter and Materials Physics.

Citation for the original published paper (version of record):

Bernad, J., Jääskeläinen, M., Zulicke, U. (2010)

Effects of a quantum measurement on the electric conductivity: Application to graphene.

Physical Review B. Condensed Matter and Materials Physics, 81 (7): 073403

http://dx.doi.org/10.1103/PhysRevB.81.073403

Access to the published version may require subscription.

N.B. When citing this work, cite the original published paper.

Permanent link to this version:

http://urn.kb.se/resolve?urn=urn:nbn:se:du-11202 


\title{
Effects of a quantum measurement on the electric conductivity: Application to graphene
}

\author{
J. Z. Bernád, ${ }^{1, *}$ M. Jääskeläinen, ${ }^{1}$ and U. Zülicke ${ }^{1,2}$ \\ ${ }^{1}$ Institute of Fundamental Sciences and MacDiarmid Institute for Advanced Materials and Nanotechnology, Massey University, \\ Manawatu Campus, Private Bag 11 222, Palmerston North 4442, New Zealand \\ ${ }^{2}$ Centre for Theoretical Chemistry and Physics, Massey University, Albany Campus, Private Bag 102904, \\ North Shore MSC, Auckland 0745, New Zealand
}

(Received 9 December 2009; published 8 February 2010)

\begin{abstract}
We generalize the standard linear-response (Kubo) theory to obtain the conductivity of a system that is subject to a quantum measurement of the current. Our approach can be used to specifically elucidate how back-action inherent to quantum measurements affects electronic transport. To illustrate the utility of our general formalism, we calculate the frequency-dependent conductivity of graphene and discuss the effect of measurement-induced decoherence on its value in the dc limit. We are able to resolve an ambiguity related to the parametric dependence of the minimal conductivity.
\end{abstract}

DOI: 10.1103/PhysRevB.81.073403

The fact that measurements exert a back-action on the measured object has attracted a lot of attention, ${ }^{1-3}$ partly due to its relevance for the foundations of quantum physics, but also because of implications for metrology ${ }^{4}$ and the design of solid-state devices. ${ }^{5}$ Fundamental considerations necessitate a distinction between selective and nonselective descriptions of quantum measurements. ${ }^{6}$ Selective descriptions use stochastic differential equations, ${ }^{7,8}$ or restricted path integrals, ${ }^{9}$ and result in conditional quantum dynamics when the measurement results are recorded. Some properties of selective measurements have been verified experimentally for areas as diverse as cavity QED (Ref. 10) and superconducting phase qubits. ${ }^{11}$ Nonselective descriptions represent the evolution of the measured system irrespective of the measurement result. This description takes into account all possible readouts, and the actual readout is assumed not to be known. ${ }^{6,8}$ Quantummechanical back-action on the unsharply measured system causes loss of coherence between eigenstates of the measured quantity. In this work, we discuss measurement backaction theoretically within the nonselective framework. This approach makes it possible to determine how a macroscopic observable such as the conductivity of a system is affected when the current is detected in an unsharp-measurement scenario. We derive a generalized Kubo formula where the measurement back-action provides a natural damping mechanism. We demonstrate the power of the developed general formalism by calculating the frequency-dependent conductivity $\sigma(\omega)$ of graphene, ${ }^{12}$ a promising candidate for future microelectronics and nanoelectronics ${ }^{13}$ and also a lowenergy laboratory of relativistic physics. ${ }^{14,15}$ Electronictransport properties of graphene were analyzed in several previous studies using different methods, e.g., the LandauerBüttiker formalism, ${ }^{16,17}$ the linear-response Kubo formula, ${ }^{17-20}$ and the Boltzmann equation. ${ }^{21}$ It was found that different parametric dependences of the dc conductivity can result from different limiting procedures applied to ordinary Kubo formulas. ${ }^{20}$ Within our generalized Kubo formalism, we obtain physical conditions for when these results apply. Two regimes can be distinguished by comparison of the energy scale $\hbar \Gamma$ that quantifies measurement-induced decoherence with the greater one among the thermal energy $k_{B} T$ and the chemical potential $\mu$ (measured from the Dirac point). Weak back-action $\left(\hbar \Gamma<\max \left\{k_{B} T, \mu\right\}\right)$ results in
PACS number(s): 72.10.Bg, 03.65.Ta, 73.23.-b, 81.05.U-

Drude-type behavior $\sigma(0) \propto 1 / \Gamma$. In the opposite (strongback-action) limit, a mixing of intraband and interband contributions occurs that changes the parametric dependence of the dc conductivity such that $\sigma(0) \propto \Gamma$.

We employ the linear-response (Kubo) formalism ${ }^{22}$ and divide the system's Hamiltonian into the part $\hat{H}_{0}$, which governs the free evolution, and $\delta \hat{H}$, the perturbation associated with an external electric field E. For simplicity, we take the latter to be constant in space and assume the field to be applied between $t=-\infty$ and $t=0$. The perturbation Hamiltonian is $\delta \hat{H}=-e \mathbf{E} \cdot \hat{\mathbf{r}} e^{i \omega t}$. In a nonselective description, ${ }^{2,3}$ the dynamics of the density matrix, when the current is measured, is governed by a master equation with the back-action caused by a term of Lindblad form ${ }^{23}$

$$
\frac{d \hat{\rho}}{d t}=-\frac{i}{\hbar}[\hat{H}, \hat{\rho}]-\frac{\gamma}{8}[\hat{j},[\hat{j}, \hat{\rho}]]=\mathcal{L} \hat{\rho}-\frac{i}{\hbar}[\delta \hat{H}, \hat{\rho}],
$$

where $\mathcal{L} \hat{\rho}=-\frac{i}{\hbar}\left[\hat{H}_{0}, \hat{\rho}\right]-\frac{\gamma}{8}[\hat{j},[\hat{j}, \hat{\rho}]]$ with the current operator $\hat{j}=i \frac{e}{\hbar}\left[\hat{H}_{0}, \hat{r}\right]$ being our measured observable. ${ }^{24}$ We note that the measured observable is arbitrarily chosen to be the current, as it is relevant for the conductance calculation at hand. The main parameter of an unsharp quantum measurement device, as described by Eq. (1), is the detection performance $\gamma=(\Delta t)^{-1}(\Delta j)^{-2}$, where $\Delta t$ is the time resolution or, equivalently, the inverse bandwidth of the detector and $\Delta j$ the statistical error characterizing unsharp detection of the average current. Within linear-response theory, we can linearize $\hat{\rho}$ $=\hat{\rho}_{0}+\delta \hat{\rho}$, where $\hat{\rho}_{0}$ is the system's equilibrium density matrix. Keeping only linear terms in Eq. (1), we get

$$
\frac{d \delta \hat{\rho}}{d t}=\mathcal{L} \delta \rho-\frac{i}{\hbar}\left[\delta \hat{H}, \hat{\rho}_{0}\right]-\frac{\gamma}{8}\left[\hat{j},\left[\hat{j}, \hat{\rho}_{0}\right]\right],
$$

assuming that the unsharp detection does not affect the equilibrium and using $\left[\hat{H}_{0}, \hat{\rho}_{0}\right]=0$ as well as $[\delta \hat{H}, \delta \hat{\rho}] \simeq 0$. Introducing $\Delta \hat{\rho}=e^{-\mathcal{L} t} \delta \hat{\rho}$ yields

$$
\frac{d \Delta \hat{\rho}}{d t}=e^{-\mathcal{L} t}\left\{-\frac{i}{\hbar}\left[\delta \hat{H}, \hat{\rho}_{0}\right]-\frac{\gamma}{8}\left[\hat{j},\left[\hat{j}, \hat{\rho}_{0}\right]\right]\right\} .
$$

Note that $\Delta \hat{\rho}$ and $\delta \hat{\rho}$ have the same value at $t=0$, and both vanish at $t=-\infty$. Integration yields 


$$
\delta \hat{\rho}(t=0)=\int_{-\infty}^{0} d t e^{-\mathcal{L} t}\left\{-\frac{i}{\hbar}\left[\delta \hat{H}, \hat{\rho}_{0}\right]-\frac{\gamma}{8}\left[\hat{j},\left[\hat{j}, \hat{\rho}_{0}\right]\right]\right\} .
$$

The exponential factor in Eq. (4) ensures convergence of the time integral, making it unnecessary to introduce the phenomenological adiabatic damping parameter employed in conventional linear-response theory. ${ }^{22}$ Inserting Eq. (4) into the expectation value for the current density $\hat{j}$ and dividing by $|\mathbf{E}|$ yields the optical conductivity

$$
\sigma_{\mu \nu}(\omega)=\int_{-\infty}^{0}\left[K_{\mu \nu}(t) e^{i \omega t}+K_{\mu \nu}^{\prime}(t)\right] d t
$$

with the kernels

$$
\begin{gathered}
K_{\mu \nu}(t)=-\frac{1}{i \hbar} \operatorname{Tr}\left\{\hat{j}_{\mu} e^{-\mathcal{L}\left(\hat{j}_{\mu}\right) t}\left(\left[e \hat{r}_{\nu}, \hat{\rho}_{0}\right]\right)\right\}, \\
K_{\mu \nu}^{\prime}(t)=\operatorname{Tr}\left(\frac{\hat{j}_{\mu}}{E_{\nu}} e^{-\mathcal{L}\left(\hat{j}_{\mu}\right) t}\left\{-\frac{\gamma}{8}\left[\hat{j}_{\mu},\left[\hat{j}_{\mu}, \hat{\rho}_{0}\right]\right]\right\}\right) .
\end{gathered}
$$

Equations (6) and (7) are the general result for the response to an unsharp measurement and could, in principle, be applied to any quantum system. As an instructive application, we now use Eq. (5) to calculate the frequencydependent conductivity of single-layer graphene within the continuum model for quasiparticles close to the $\mathbf{K}$ point in the Brillouin zone. In Eq. (1), the Hamiltonian $\hat{H}_{0}$ could, in principle, include Coulomb interactions, impurity scattering, density dependencies, etc. In the present work, we just use the free-quasiparticle Hamiltonian for graphene in planewave representation,

$$
\hat{H}_{0}(\mathbf{k})=\hbar v\left(\sigma_{x} k_{x}+\sigma_{y} k_{y}\right),
$$

where $k_{x}$ and $k_{y}$ are the Cartesian components of wave vector $\mathbf{k}, \sigma_{i}$ denote Pauli matrices acting in the sublattice-related pseudospin space, and $v$ is the Fermi velocity, which has a value $\simeq 10^{6} \mathrm{~m} / \mathrm{s}$. With the position operator $\hat{\mathbf{r}}$ being the wave-vector gradient, the current operator is $\hat{j}_{\mu}$ $=\frac{i e}{\hbar}\left[\hat{H}_{0}(\mathbf{k}), \hat{r}_{\mu}\right]=\frac{e}{\hbar} \frac{\partial \hat{H}_{0}(\mathbf{k})}{\partial k_{\mu}}$. Single-particle eigenstates of clean graphene can be written as a direct product of a plane wave in configuration space with a spinor $|n\rangle=|\mathbf{k}\rangle \otimes|\sigma\rangle_{\mathbf{k}}$. Here $\sigma$ labels the electron and hole bands, respectively, and the spinor wave function depends on wave vector $\mathbf{k}$. The current operators with the Hamiltonian (8) in the spinor space are $\hat{j}_{x}=e v \sigma_{x}$, and $\hat{j}_{y}=e v \sigma_{y}{ }^{25}$ From the definition of the equilibrium density matrix in the spinor space we find $\hat{\rho}_{0}|\sigma\rangle_{\mathbf{k}}$ $=f\left(\hbar \epsilon_{\mathbf{k}, \sigma}\right)|\sigma\rangle_{\mathbf{k}}$, and $\hat{H}_{0}|\sigma\rangle_{\mathbf{k}}=\hbar \epsilon_{\mathbf{k}, \sigma}|\sigma\rangle_{\mathbf{k}}$, where $f$ is the FermiDirac distribution function and $\epsilon_{\mathbf{k}, \pm}= \pm|\mathbf{k}|$. (For simplicity, the speed $v$ has been absorbed into k.) Together with the antisymmetry in momentum space, this implies $K_{\mu \nu}^{\prime}=0$. The calculation of $K_{\mu \nu}$ is straightforward, and using the Laplace transform to solve for the dynamics, we find

$$
K_{\mu \nu}(t)=\frac{e^{2}}{\hbar} \int \frac{d^{2} \mathbf{k}}{(2 \pi)^{2}} \operatorname{Res}\left\{\tilde{K}_{\mu \nu}(\mathbf{k}, \gamma, z) e^{z t}\right\},
$$

where Res stands for the sum of residues of the integrand. It can be seen that $K_{x y}=K_{y x}=0$. The remaining two conductivities are identical, as a change in variables $k_{x} \leftrightarrow k_{y}$ in the expression for $K_{x x}$ yields $K_{y y}$. The choice of conductivity measured will decide what kind of back-action will influence the system. Here we take $\hat{j}_{x}$, the current along the $x$ direction, which breaks the isotropy of the problem and we find

$$
\tilde{K}_{x x}(\mathbf{k}, \Gamma, z)=\frac{k_{x}^{2}|\mathbf{k}|\left(16 \Gamma^{2}-8 z \Gamma+4|\mathbf{k}|^{2}+z^{2}\right) \sum_{\sigma}\left(-\left.\frac{d f(\hbar \epsilon)}{d \epsilon}\right|_{\epsilon=\epsilon_{\mathbf{k}, \sigma}}\right)+k_{y}^{2}(z-4 \Gamma)^{2}\left[f\left(\hbar \epsilon_{\mathbf{k},-}\right)-f\left(\hbar \epsilon_{\mathbf{k},+}\right)\right]}{|\mathbf{k}|^{3}\left[16 z \Gamma^{2}-8\left(2 k_{y}^{2}+z^{2}\right) \Gamma+z\left(4|\mathbf{k}|^{2}+z^{2}\right)\right]},
$$

where the parameter $\Gamma=\gamma e^{2} v^{2} / 8$ was introduced. The cubic factor in the denominator of Eq. (10) has three roots $z_{i}$, which give the poles in Eq. (9).

For small $\Gamma$, the roots are to lowest order $z_{1}=0$ and $z_{2,3}$ $= \pm 2 i|\mathbf{k}|$. Using this and performing the time integration in the limit $\Gamma \rightarrow 0$, the known intraband and interband contributions

$$
\begin{aligned}
\frac{\sigma^{(i n t r a)}}{\sigma_{0}} & =\frac{\pi}{2} \delta(\omega) \int_{0}^{\infty} x\left[\sum_{\sigma= \pm}\left(-\left.\frac{d f(\hbar \epsilon)}{d \epsilon}\right|_{\epsilon=\sigma x}\right)\right] d x \\
& =\frac{\pi}{2} \delta\left(\frac{\hbar \omega}{k_{B} T}\right)\left[2 \log \left(1+e^{\mu / k_{B} T}\right)-\frac{\mu}{k_{B} T}\right]
\end{aligned}
$$

$$
\frac{\sigma^{(i n t e r)}}{\sigma_{0}}=\frac{\pi}{8} \frac{\sinh \left(\frac{\hbar \omega}{2 k_{B} T}\right)}{\cosh \left(\frac{\mu}{k_{B} T}\right)+\cosh \left(\frac{\hbar \omega}{2 k_{B} T}\right)}
$$

to the conductivity of clean graphene are found. The scale factor $\sigma_{0}=4 e^{2} / h$ accounts for spin and pseudospin degeneracies.

In the following, the conductivity is calculated numerically for finite values of $\Gamma$ from Eq. (5) with Eqs. (9) and (10). We use $k_{B} T$ as unit of energy. Figures 1 and 2 show the ac (optical) conductivity, ${ }^{27}$ whereas Fig. 3 shows the dc conductivity that is measured, e.g., in mesoscopic transport experiments. 


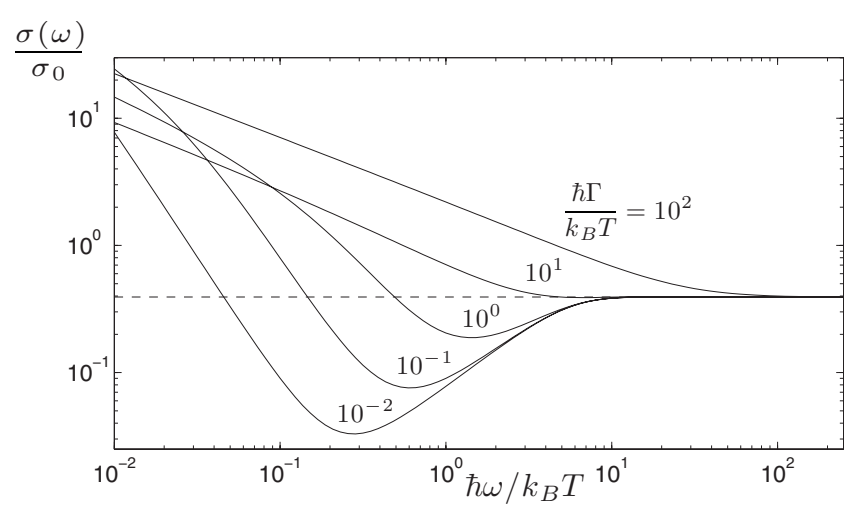

FIG. 1. Frequency dependence of the conductivity of clean single-layer graphene when the current is unsharply quantum measured. The chemical potential is fixed at $\mu / k_{B} T=1$, and the value for measurement-induced decoherence assumed for each curve is indicated. The dashed line is at $\pi / 8$. As the coupling to the measurement device is decreased, the curves more closely resemble the result found for clean graphene.

In Fig. 1, the conductivity is shown as function of frequency for different values of the coupling strength when the chemical potential remains fixed. For high frequencies, the conductivity saturates to the universal value $\pi / 8$, indicated by a dashed line. The detailed shape of the crossover to saturation depends on $\Gamma$ with higher values pushing it to higher frequencies.

In the limit of small $\Gamma$, measurement-induced decoherence appears to simulate the effect of life-time broadening due to inelastic scattering, ${ }^{19,20,26}$ but a closer look reveals that it is fundamentally different. Technically, the effect of $\Gamma$ goes beyond merely broadening of distribution functions, it also moves the position of their peaks in energy, thus changing the resonance condition. For clean graphene, there is only a delta function peak for the intraband contribution, whereas here the intraband and interband contributions to the conductivity become mixed. The existence of such a mixing has been inferred from recent experiments. ${ }^{27}$ The dependence on the chemical potential is illustrated in Fig. 2. Generally, increasing the chemical potential shifts the frequency beyond which the conductivity attains its universal saturation to

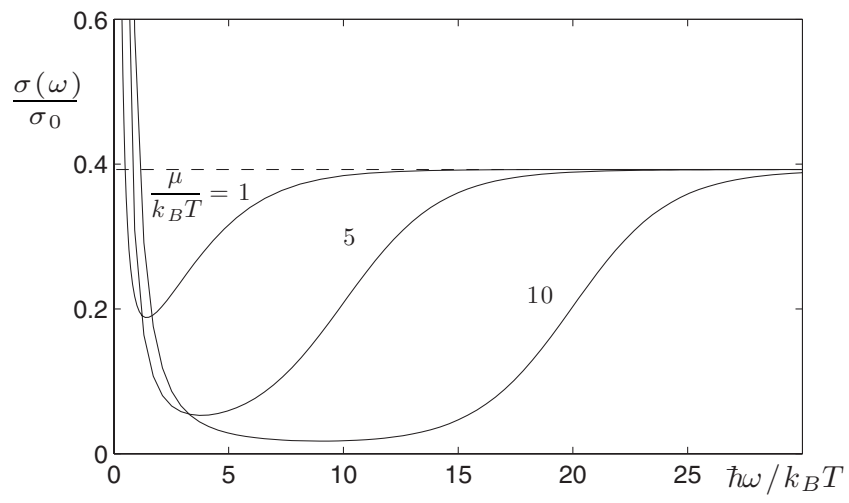

FIG. 2. ac conductivity of single-layer graphene, for fixed $\hbar \Gamma / k_{B} T=1$ and different values of chemical potential $\mu$ (measured from the Dirac point).
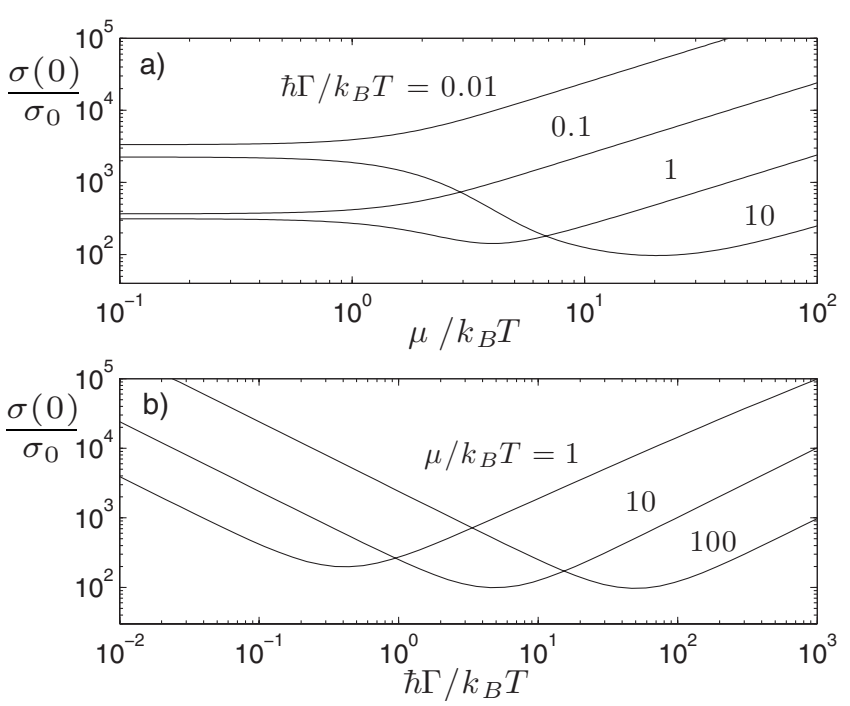

FIG. 3. (a) de conductivity plotted as a function of chemical potential for different values of $\Gamma$. The conductivity is linear in the chemical potential for large values of $\mu / k_{B} T$. (b) dc conductivity shown as a function of $\hbar \Gamma / k_{B} T$ for different values of the chemical potential. For $\mu>k_{B} T$ and $\hbar \Gamma<\mu$, the conductivity is inversely proportional to the effective rate of decoherence, in analogy with the behavior expected from inelastic impurity scattering. In contrast, for $\hbar \Gamma>\mu$ there is direct proportionality. This behavior was exhibited as long as $\mu \gtrsim k_{B} T$, whereas curves for $\mu \leq k_{B} T$ practically coincide.

higher values. This behavior is as expected theoretically ${ }^{26}$ and observed in experiments. ${ }^{27}$ At frequencies $\omega$ smaller than a few times the chemical potential, there is a significant departure from the universal conductance plateau. For fixed $\mu$ and $k_{B} T$ the saturation point in scattering models is independent of the scattering parameter, whereas in our work it strongly depends on the value of $\Gamma$. For $\mu \gtrsim \hbar \Gamma$, the saturation occurs as in clean graphene, whereas the opposite case gives saturation for larger frequencies with increasing $\Gamma$, as seen in Fig. 1.

When the effect of disorder is modeled conventionally by a life-time broadening due to inelastic scattering, ${ }^{19,20}$ a Drude peak is found for $\hbar \omega / k_{B} T \sim 0$, with a height inversely proportional to the inelastic-scattering rate. Since the master equation describing the effect of quantum measurements is formally identical to certain models of decoherence, we expect that $\sigma(0) / \sigma_{0} \sim 1 / \Gamma$. This turns out to be correct only for $\hbar \Gamma<\max \left\{\mu, k_{B} T\right\}$, as can be seen in Fig. 3(b). In the opposite limit, the parametric dependence of the dc conductivity is changed; it then grows linearly with $\Gamma$. A similar anomalous behavior was obtained in Ref. 20 by applying an unconventional limiting procedure within the Kubo formalism. Here we are able to readily identify the regimes where ordinary Drude behavior or anomalous band-mixing behavior will be exhibited. Clearly, observing the latter should be easiest when the chemical potential is at the Dirac point and the temperature is low enough to satisfy $\hbar \Gamma>k_{B} T$, which corresponds to the minimal conductivity regime for graphene. Figure 3(a) shows how the nonmonotonic $\Gamma$ dependence of the conductivity would be manifested in a typical transport experiment where the chemical potential (i.e., the density) is varied. 
The theory presented here is based on an unsharp measurement of the current density. To estimate the magnitude $\Gamma$ of measurement-induced decoherence, we must consider the two situations most closely related to our result, optical conductivity measurements, and mesoscopic transport measurements. For the latter case, it can be easily seen that $e^{2} v^{2} /(\Delta j)^{2}$ is the signal-to-noise ratio. Using typical values $\Delta \nu \gtrsim 10^{6} \mathrm{~Hz}$ for the bandwidth and $\Delta I / I \approx 10^{-3}$ for the signal-to-noise ratio, we find that values up to $\hbar \Gamma / k_{B} T$ $\approx 10^{-1}-10^{2}$ can be achieved for $T \approx 1-300 \mathrm{~K}$. For measurements of the optical conductivity, the situation is more complex, as the measured signal is induced by the currents generated by the applied optical field. As a result, additional uncertainties such as geometrical factors and detector efficiencies become important, possibly bringing down the detection performance, but this can, in principle, be compensated by the increase in bandwidth offered by optical detectors, $\Delta \nu \gtrsim 10^{9} \mathrm{~Hz}$.
In conclusion, we derived a new Kubo formula to study the effect of measurement-induced back-action on the conductivity. The back-action naturally introduces a source of damping and thus makes the converged adiabaticity parameter frequently used in Kubo formula calculations superfluous. We applied this approach to calculate the electric conductivity of single-layer graphene. Mixing of the intraband and interband contributions to the dc conductivity strongly affect its parametric dependence on the detector performance $\Gamma$. The regime of weak coupling to the measuring device models a standard Drude-type behavior, whereas in the opposite limit of strong back-action, we find that measuring a current in graphene will actually enhance the conductivity.

This work was supported by the Massey University Research Fund and by the Marsden Fund Council (Contract No. MAU0702) from Government funding, administered by the Royal Society of New Zealand. *j.z.bernad@massey.ac.nz

${ }^{1}$ V. B. Braginsky and F. Ya. Khalili, Quantum Measurement (Cambridge University Press, Cambridge, 1992).

${ }^{2}$ L. Diósi, in Encyclopedia of Mathematical Physics, edited by J.-P. Françoise, G. L. Naber, and S. T. Tsou (Academic, Oxford, 2006), Vol. 4, p. 276.

${ }^{3}$ K. Jacobs and D. A. Steck, Contemp. Phys. 47, 279 (2006).

${ }^{4}$ J. M. Geremia et al., Phys. Rev. Lett. 91, 250801 (2003).

${ }^{5}$ J. Z. Bernad et al., Phys. Rev. B 77, 073311 (2008).

${ }^{6}$ M. B. Menskii, Phys. Usp. 41, 923 (1998); 46, 1163 (2003).

${ }^{7}$ N. Gisin, Phys. Rev. Lett. 52, 1657 (1984); A. Barchielli, Phys. Rev. A 34, 1642 (1986); L. Diosi, Phys. Lett. A 129, 419 (1988).

${ }^{8}$ C. M. Caves and G. J. Milburn, Phys. Rev. A 36, 5543 (1987).

${ }^{9}$ M. B. Mensky, Phys. Rev. D 20, 384 (1979); C. M. Caves, ibid. 33, 1643 (1986).

${ }^{10}$ C. Guerlin et al., Nature (London) 448, 889 (2007).

${ }^{11}$ N. Katz et al., Science 312, 1498 (2006).

${ }^{12}$ K. S. Novoselov et al., Science 306, 666 (2004); K. S. Novoselov et al.,Proc. Natl. Acad. Sci. U.S.A. 102, 10451 (2005).

${ }^{13}$ P. Avouris et al., Nat. Nanotechnol. 2, 605 (2007); Z. Chen et al., Physica E 40, 228 (2007); J. B. Oostinga et al., Nature Mater. 7, 151 (2007).

${ }^{14}$ Y. Zhang et al., Phys. Rev. Lett. 94, 176803 (2005); Y. Zhang et al., Nature (London) 438, 201 (2005).

${ }^{15}$ K. S. Novoselov et al., Nature (London) 438, 197 (2005).

${ }^{16}$ M. I. Katsnelson, Eur. Phys. J. B 51, 157 (2006); J. Tworzydlo et al., Phys. Rev. Lett. 96, 246802 (2006).

${ }^{17}$ S. Ryu et al., Phys. Rev. B 75, 205344 (2007).

${ }^{18}$ A. W. W. Ludwig et al., Phys. Rev. B 50, 7526 (1994); E. V. Gorbar et al., ibid. 66, 045108 (2002); V. P. Gusynin and S. G. Sharapov, Phys. Rev. Lett. 95, 146801 (2005); Phys. Rev. B 73,
245411 (2006); N. M. R. Peres et al.,ibid. 73, 125411 (2006); P. M. Ostrovsky et al., ibid. 74, 235443 (2006); J. Cserti, ibid. 75, 033405 (2007).

${ }^{19}$ K. Ziegler, Phys. Rev. Lett. 97, 266802 (2006).

${ }^{20}$ K. Ziegler, Phys. Rev. B 75, 233407 (2007).

${ }^{21}$ K. Nomura and A. H. MacDonald, Phys. Rev. Lett. 96, 256602 (2006); 98, 076602 (2007); N. M. R. Peres et al., Phys. Rev. B 76, 073412 (2007); E. H. Hwang et al., Phys. Rev. Lett. 98, 186806 (2007)

${ }^{22}$ O. Madelung, Introduction to Solid-State Theory (Springer, Berlin, 1978).

${ }^{23}$ Starting from positive operator-valued measure and using the standard Gaussian model we arrive in the selective measurement case to stochastic master equation and in the nonselective case at a master equation with the double commutator.

${ }^{24}$ For other types of experiments, the corresponding measured operator will occur in the Lindbladian term and, thus, induce backaction on the system. Although the formalism is, in its mathematical structure, identical to decoherence, the effects on the dynamics depend on what kind of measurement is performed, which is a matter of choice. In contrast, environmentally induced decoherence that is determined by interaction with an ever-present reservoir cannot be manipulated at will. The choice of measured observable thus decides the type of decoherence present in the system through experimental design.

${ }^{25}$ The Hilbert space is decomposed into irreducible components. Each pseudospin sector is a superselection sector, preserved by the current operator.

${ }^{26}$ N. M. R. Peres et al., EPL 84, 38002 (2008).

${ }^{27}$ K. F. Mak et al., Phys. Rev. Lett. 101, 196405 (2008); F. Wang et al., Science 320, 206 (2008); Z. Q. Li et al., Nat. Phys. 4, 532 (2008). 